\title{
High-Dose Cyclophosphamide Therapy Associated with Diffuse Alveolar Hemorrhage after Allogeneic Hematopoietic Stem Cell Transplantation
}

\author{
Xiao-Dong Mo Lan-Ping Xu Dai-Hong Liu Xiao-Hui Zhang Huan Chen \\ Yu-Hong Chen Wei Han Yu Wang Feng-Rong Wang Jing-Zhi Wang \\ Kai-Yan Liu Xiao-Jun Huang \\ Beijing Key Laboratory of Hematopoitic Stem Cell Transplantation, Peking University People's Hospital and \\ Institute of Hematology, Beijing, PR China
}

\section{Key Words}

Diffuse alveolar hemorrhage · Transplantation •

Cyclophosphamide $\cdot$ Corticosteroid

\begin{abstract}
Background: The occurrence of diffuse alveolar hemorrhage (DAH) after allogeneic hematopoietic stem cell transplantation (allo-HSCT) is rare but severe. There are few reports that have examined the correlation between pre-HSCT chemotherapeutic exposure and DAH. Objectives: We examine the role of pre-HSCT chemotherapeutic exposure, conditioning regimens, pre-HSCT comorbidities and transplant-related complications in the development of DAH after allo-HSCT and evaluate the effect of the high-dose corticosteroid strategy on DAH. Methods: A retrospective nested case-control study was designed. Cases with DAH and controls matched for year of allo-HSCT and length of follow-up were identified from a cohort of 597 patients who underwent allo-HSCT between 2006 and 2011 for acute leukemia. Results: Twenty-two patients suffered from DAH; the mean age at the time of presentation was 30.4 years $( \pm 12.9)$ and the mean time to presentation was 7.8 months ( \pm 8.1 ) postHSCT. The pre-HSCT cyclophosphamide exposure and the cumulative cyclophosphamide dose were significantly high-
\end{abstract}

er among the DAH cases compared with the controls, and the cumulative cyclophosphamide dose of $\geq 5 \mathrm{~g} / \mathrm{m}^{2}$ was independently associated with $\mathrm{DAH}(\mathrm{OR}=3.4, \mathrm{p}=0.030)$. Highdose corticosteroid treatment did not significantly improve survival. Conclusions: From these results we can identify patients who are at a higher risk of developing DAH after allo$\mathrm{HSCT}$, and we found that high-dose corticosteroid therapy may not alter the poor outcome associated with this syndrome.

Copyright $\odot 2012$ S. Karger AG, Basel

\section{Introduction}

Allogeneic hematopoietic stem cell transplantation (allo-HSCT) is an effective treatment for hematological malignancies and many patients are cured or achieve long-term remissions. However, pulmonary complications after allo-HSCT can seriously influence the survival of patients. Diffuse alveolar hemorrhage (DAH) is found in $2-14 \%$ of patients receiving HSCT [1-7] and it is considered as one of the most severe pulmonary complications. DAH is associated with a $60-83 \%$ mortality rate $[1-3,6-8]$; therefore, it is important to pay more attention to this complication.

\section{KARGER}

E-Mail karger@karger.com www.karger.com/res
(C) $2012 \mathrm{~S}$. Karger AG, Basel

$0025-7931 / 12 / 0866-0453 \$ 38.00 / 0$

Accessible online at: www.karger.com/res
Xiao-Jun Huang

Peking University People's Hospital and Institute of Hematology Beijing Key Laboratory of Hematopoitic Stem Cell Transplantation No. 11, South Street of Xizhimen, Beijing 100044 (PR China)

E-Mail huangxiaojun@bjmu.edu.cn 
The etiology of DAH in the HSCT recipient remains largely unknown and previous studies found that several factors may be associated with the development of DAH after allo-HSCT. These factors include older age, preHSCT forced expiratory volume in $1 \mathrm{~s}\left(\mathrm{FEV}_{1}\right)$ of $\leq 80 \%$, allogeneic donor source, myeloablative conditioning regimen, previous history of HSCT, cyclosporine/methotrexate as graft-versus-host disease (GVHD) prophylaxis, severe acute GVHD and thrombotic microangiopathy [1, $4,5,9,10]$. However, none of them were well recognized. Moreover, there are few reports that have examined the correlation between pre-HSCT chemotherapeutic exposure and DAH. Among the patients suffering from DAH after allo-HSCT, 30-75\% were diagnosed with acute leukemia before HSCT $[3,4,6]$, which suggests that chemotherapy may be correlated with DAH. Raptis et al. [4] reported that high-dose chemotherapy which induced temporary marrow aplasia was significantly associated with $\mathrm{DAH}$; however, the small sample size of DAH patients $(n=4)$ and the broad definition of high-dose chemotherapy makes it difficult to further evaluate the relationship between chemotherapy and DAH after allo-HSCT. Among the patients with hematologic malignancies, the associations between the types and cumulative doses of pre-HSCT chemotherapeutic agents and DAH may be more important. Confirming these associations can help us to determine which patients are at risk for $\mathrm{DAH}$; in this case, the patients should be given a more reasonable conditioning regimen and monitored closely. This is an important preventive measure to protect patients against DAH after allo-HSCT, but very little research has examined this aspect.

Thus, the major objectives of our study were to examine the independent roles of pre-HSCT chemotherapeutic exposures, conditioning regimens, pre-HSCT comorbidities and transplant-related complications in the development of DAH after allo-HSCT, and to evaluate the effect of high-dose corticosteroid therapy on DAH.

\section{Materials and Methods}

\section{Patients}

A retrospective nested case-control study was designed. The cases were identified from a cohort of 597 patients who underwent allo-HSCT for acute leukemia between 2006 and 2011 at the Institute of Hematology, Peking University. The patients who had developed DAH after HSCT were eligible. Using 'risk-set sampling' (for each DAH case, a set of controls of the case was selected from subjects at risk at the time of DAH occurrence, and the matching in this design allows for tight control of the confounding effects of time in the analysis) [11], we randomly selected con- trols in a 3:1 ratio with matching for year of the HSCT ( \pm 2 years) and the length of follow-up ( \pm 2 months). A total of 22 patients with DAH and 66 matched controls were included in the analyses. The mean follow-up period after the HSCT was 8.5 months $( \pm 8.0)$ for the DAH cases and 7.0 months $( \pm 6.1)$ for the controls $(\mathrm{p}=$ 0.344 ). The characteristics of the DAH cases and controls are summarized in table 1 . The study protocol was approved by the ethics committee at Peking University People's Hospital. Informed consent was provided according to the Declaration of Helsinki.

\section{Donor Selection}

A human leukocyte antigen (HLA)-identical sibling donor (ISD) was the first choice for allo-HSCT. If an ISD was unavailable as a first-treatment option, patients without a suitable closely HLA-matched unrelated donor, that is, with more than 8 out of 10 matching HLA-A, B, C, DR and DQ loci and at least 5 out of 6 matching HLA-A, B and DR loci, or whose disease status left insufficient time for an unrelated donor search, were eligible for HLA-partially matched related donor (PMRD) HSCT. To determine HLA-A and HLA-B status, low-resolution DNA techniques were used. High-resolution techniques were used for HLA-DRB1 typing. All donor-recipient pairs were typed at the HLA-A, B and DR loci at our institute. Each patient with PMRD received stem cells from a family member who shared 1 HLA haplotype with the patient but differed to a variable degree for the HLA-A, B and D antigens of the haplotype not shared. Apart from each donor recipient pair, HLA typing was performed for parents and offspring to be strictly analyzed to guarantee true haploid genetic background. The HLA disparities in the PMRD cohort are shown in table 1 .

\section{Transplantation Regimen}

The preconditioning regimen consisted of cytarabine, busulfan ( $4 \mathrm{mg} / \mathrm{kg} /$ day, -8 to -6 days), cyclophosphamide $\left(1.8 \mathrm{~g} / \mathrm{m}^{2} /\right.$ day, -5 to -4 days) and simustine (Me-CCUN, $250 \mathrm{mg} / \mathrm{m}^{2},-3$ days). Cytarabine was administered at $4 \mathrm{~g} / \mathrm{m}^{2} /$ day ( -10 to -9 days) to the PMRD HSCT groups at $2 \mathrm{~g} / \mathrm{m}^{2} / \mathrm{day}$ ( -10 to -9 days) to the matched unrelated donor HSCT groups, and at $2 \mathrm{~g} / \mathrm{m}^{2} / \mathrm{d}$ ( -9 days) to the ISD HSCT group. Rabbit antithymocyte globulin (Thymoglobulin, $2.5 \mathrm{mg} / \mathrm{kg},-5$ to -2 days; Sanofi, France) was used in the PMRD group. A cyclophosphamide and antithymocyte globulin regimen was used for patients with severe aplastic anemia. Cyclophosphamide was replaced with fludarabine in the patients who were $\geq 55$ years of age and/or had ECOG scores of $\geq 2(n=8)$. The prevention regimen for GVHD was cyclosporine A + short-course methotrexate + mycophenolate mofetil [12]. Standard-risk patients were defined as patients in the first or second complete remission of acute myeloid leukemia (AML) and Philadelphia chromosome-negative acute lymphoblastic leukemia (ALL). The other patients were classified as high risk.

\section{Diagnosis and Treatment of DAH}

Detailed clinical information on the patients was gathered from the transplant program database and if there were unexpected gaps in a patient's history, we contacted the caregivers or called the patient directly. DAH was diagnosed according to the following criteria: (1) acute hypoxemic respiratory failure, defined as an oxygenation index (arterial partial pressure of oxygen/ fraction of inspiration oxygen ratio) $<200 \mathrm{~mm} \mathrm{Hg}$, which re- 
Table 1. Patient characteristics

\begin{tabular}{|c|c|c|c|}
\hline & 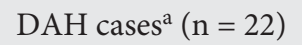 & Controls $^{\mathrm{a}}(\mathrm{n}=66)$ & $\mathrm{p}$ \\
\hline \multicolumn{4}{|l|}{ Gender } \\
\hline Male & $18(81.8)$ & $50(75.8)$ & 0.557 \\
\hline Female & $4(18.2)$ & $16(24.2)$ & \\
\hline Age at HSCT, years & $30.4 \pm 12.9$ & $30.2 \pm 11.9$ & 0.968 \\
\hline Smoking pre-HSCT & $6(27.3)$ & $13(19.7)$ & 0.551 \\
\hline \multicolumn{4}{|l|}{ Pulmonary function test before HSCT } \\
\hline $\mathrm{FEV}_{1}, \%$ & $91.9 \pm 15.5$ & $91.3 \pm 14.1$ & 0.883 \\
\hline $\mathrm{FEV}_{1} / \mathrm{FVC}, \%$ & $85.5 \pm 6.3$ & $86.4 \pm 7.1$ & 0.628 \\
\hline \multicolumn{4}{|l|}{ Diagnosis } \\
\hline AML & $11(50.0)$ & $39(59.1)$ & 0.456 \\
\hline ALL & $11(50.0)$ & $27(40.9)$ & \\
\hline \multicolumn{4}{|l|}{ Status at HSCT } \\
\hline Standard risk & $18(81.8)$ & $58(87.9)$ & 0.485 \\
\hline High risk & $4(18.2)$ & $8(12.1)$ & \\
\hline \multicolumn{4}{|l|}{ Donor match } \\
\hline HLA-identical sibling donor & $5(22.7)$ & $18(27.3)$ & 0.790 \\
\hline HLA-partially matched related donor & $16(72.7)$ & $42(63.6)$ & \\
\hline HLA-matched unrelated donor & $1(4.6)$ & $6(9.1)$ & \\
\hline \multicolumn{4}{|l|}{ HLA-A, -B, -DR mismatched } \\
\hline 0 & $6(27.3)$ & $24(36.4)$ & 0.239 \\
\hline 1 & $3(17.6)$ & $2(3.0)$ & \\
\hline 2 & $6(27.3)$ & $14(21.2)$ & \\
\hline 3 & $7(31.8)$ & $26(39.4)$ & \\
\hline \multicolumn{4}{|l|}{ Source of stem cell } \\
\hline Bone marrow and peripheral blood & $19(86.4)$ & $58(87.9)$ & 0.852 \\
\hline Peripheral blood & $3(13.6)$ & $8(12.1)$ & \\
\hline \multicolumn{4}{|l|}{ HCT-CI scores } \\
\hline Low risk & $4(18.2)$ & $18(27.3)$ & 0.543 \\
\hline Intermediate risk & $6(27.3)$ & $21(31.8)$ & \\
\hline High risk & $12(54.5)$ & $27(40.9)$ & \\
\hline
\end{tabular}

Values are presented as $\mathrm{n}(\%)$ or mean $\pm \mathrm{SD} . \mathrm{FVC}=$ Forced vital capacity; HCT-CI = hematopoietic cell transplantation-specific comorbidity index.

${ }^{a}$ Group matching criteria included year of HSCT ( \pm 2 years) and duration of follow-up ( \pm 2 months).

quired supplemental oxygen or mechanical ventilation support; (2) multilobar pulmonary infiltrates observed on chest radiographs; (3) bronchoalveolar lavage (BAL) showing progressively bloodier returns from three or more separate subsegmental bronchi, and/or the presence of $20 \%$ or more hemosiderin-laden macrophages; (4) no clear bacterial, viral or fungal pathogens detected in the BAL fluid, and (5) no evidence of cardiogenic pulmonary edema based on clinical findings, central venous pressure measurements or cardiac echocardiography $[8,9,13]$.

All the DAH patients received supportive treatment, including oxygen support and/or mechanical ventilation, maintenance of fluid and electrolyte balance, and supplements of coagulation factors or palate. Prophylactic or empiric antimicrobial agents were used as clinically indicated. The treatments with high-dose corticosteroids were typically initiated with methylprednisolone $250 \mathrm{mg}$ to $2 \mathrm{~g}$ IV daily for the first 4-5 days and then gradually tapering off over the next 2-4 weeks. To more precisely evaluate the dose of corticosteroids, we recalculated the dose they received based on body surface area and the cases were further divided into two groups based on the initial dosage of daily methylprednisolone (MP): the high-dose group received MP $\geq 160 \mathrm{mg} / \mathrm{m}^{2} / \mathrm{day}$, and the low-dose group received $\mathrm{MP}<160$ $\mathrm{mg} / \mathrm{m}^{2} /$ day. We used $160 \mathrm{mg} / \mathrm{m}^{2}$ as the dividing point for our analysis because it represented the median initial dosage of MP for DAH patients in our cohort. Symptoms and the oxygenation index were evaluated daily. Abatements of dyspnea, coughing and hemoptysis were considered to be symptom improvement, and contrariwise to be symptom deterioration. The oxygenation index increase of $100 \mathrm{~mm} \mathrm{Hg}$ or more was considered as an improvement. No patients received human recombinant activated factor VIIa in our study.

\section{Microbiological Techniques}

Bacterial and fungal cultures, and viral antigen studies to test for cytomegalovirus (CMV), adenovirus, respiratory syncytial virus and influenza A antigen were conducted on the BAL fluid. 
Table 2. Characteristics of pre-HSCT chemotherapeutic exposure and conditioning regimen

\begin{tabular}{|c|c|c|c|}
\hline & DAH $\operatorname{cases}^{\mathrm{a}}(\mathrm{n}=22)$ & Controls $^{\mathrm{a}}(\mathrm{n}=66)$ & $\mathrm{p}$ \\
\hline Pre-HSCT courses of chemotherapy & $5.0 \pm 1.0$ & $4.7 \pm 1.1$ & 0.285 \\
\hline \multicolumn{4}{|l|}{ Pre-HSCT chemotherapy } \\
\hline Cyclophosphamide, $\mathrm{g} / \mathrm{m}^{2}$ & $1.5 \pm 1.8$ & $0.7 \pm 1.1$ & $0.044^{*}$ \\
\hline Anthracycline, $\mathrm{mg} / \mathrm{m}^{2}$ & $145.4 \pm 105.7$ & $131.5 \pm 68.7$ & 0.480 \\
\hline Etoposide, $\mathrm{mg} / \mathrm{m}^{2}$ & $274.5 \pm 120.7$ & $348.2 \pm 159.4$ & 0.305 \\
\hline Cytarabine, $\mathrm{g} / \mathrm{m}^{2}$ & $9.5 \pm 7.2$ & $12.2 \pm 11.1$ & 0.337 \\
\hline Methotrexate, $\mathrm{g} / \mathrm{m}^{2}$ & $2.3 \pm 1.3$ & $2.3 \pm 1.6$ & 0.941 \\
\hline \multicolumn{4}{|l|}{ Pre-HSCT chemotherapy ${ }^{\mathrm{b}}$} \\
\hline Cyclophosphamide $\geq 3.0 \mathrm{~g} / \mathrm{m}^{2}$ & $6(27.3)$ & $4(6.1)$ & $0.014^{*}$ \\
\hline \multicolumn{4}{|l|}{ Conditioning regimens } \\
\hline Cyclophosphamide, $\mathrm{g} / \mathrm{m}^{2}$ & $3.4 \pm 0.8$ & $3.2 \pm 1.1$ & 0.282 \\
\hline Busulfan, $\mathrm{mg} / \mathrm{m}^{2}$ & $357.5 \pm 36.9$ & $356.6 \pm 51.7$ & 0.939 \\
\hline Cytarabine, $\mathrm{g} / \mathrm{m}^{2}$ & $5.8 \pm 2.7$ & $5.9 \pm 2.7$ & 0.817 \\
\hline \multicolumn{4}{|l|}{ Cumulative chemotherapeutic dose $\mathrm{c}^{\mathrm{c}}$} \\
\hline Cyclophosphamide, $\mathrm{g} / \mathrm{m}^{2}$ & $4.9 \pm 2.0$ & $3.8 \pm 1.6$ & $0.028^{*}$ \\
\hline Cytarabine, $\mathrm{g} / \mathrm{m}^{2}$ & $13.5 \pm 8.8$ & $17.0 \pm 11.2$ & 0.191 \\
\hline \multicolumn{4}{|l|}{ Cumulative chemotherapy ${ }^{\mathrm{b}}$} \\
\hline Cyclophosphamide $\geq 5.0 \mathrm{~g} / \mathrm{m}^{2}$ & $10(45.5)$ & $14(21.2)$ & $0.027^{*}$ \\
\hline \multicolumn{4}{|c|}{$\begin{array}{l}\text { Values are presented as } \mathrm{n}(\%) \text { or mean } \pm \mathrm{SD} .{ }^{*} \mathrm{p}<0.05 \text {. } \\
\text { a Group matching criteria included year of HSCT }( \pm 2 \text { years) and duration of follow-up ( } \pm 2 \text { months). } \\
\text { b The } 75 \text { th percentile was used as the cutoff point. } \\
\text { c The cumulative doses of cyclophosphamide and cytarabine included the pre-HSCT exposure and doses } \\
\text { ed in conditioning regimens. }\end{array}$} \\
\hline
\end{tabular}

Blood cultures for bacteria and fungi were performed on patients with fever and real-time quantitative PCR for detection of CMVDNA was used to detect CMV antigenemia every week. The appearance of any identifiable infection 1 week after the diagnosis of DAH was defined as a secondary infection.

\section{Pre-HSCT Exposure Variables}

The exposure variables included the demographics (gender, age at the time of the HSCT, smoking history and pulmonary function test), disease characteristics (diagnosis and stage), treatment before the HSCT (pre-HSCT cycles of chemotherapy and pre-HSCT exposure of common chemotherapeutic agents) and transplant characteristics (donor type, stem cell source and conditioning regimens).

\section{Cumulative Doses of Chemotherapeutic Agents}

The cumulative dose of anthracyclines was calculated as the equivalent dose of native doxorubicin [14] and the cumulative doses of cyclophosphamide and cytarabine included the preHSCT exposure and doses used in conditioning regimens.

\section{Pre-HSCT Comorbidities and Severe Transplant-Related Complications}

Hematopoietic cell transplantation-specific comorbidity index (HCT-CI) was used to evaluate the severity of pre-HSCT comorbidities at the time of transplantation and it was separated into 3 risk groups: low (0), intermediate (1-2) and high ( $\geq 3)$ [15].
Severe transplant-related complications included acute GVHD $\geq$ grade III, chronic GVHD $\geq$ the moderate category, hepatic sinusoidal obstruction syndrome, engraftment syndrome, thrombotic microangiopathy and post-transplantation lymphoproliferative disease. These complications were diagnosed and graded in accordance with common international criteria [16-20]. Transplant-related complications should have developed before the onset of DAH in cases or for a comparable period of follow-up in controls.

\section{Statistical Analysis}

The duration from DAH to death represents the period from DAH diagnosis to death, and transplantation survival represents the period from stem cell infusion (day 0) to the date of the last follow-up or death. The demographics, pre-HSCT chemotherapeutic exposure (using the 75th percentile as the cutoff point), HCT-CI scores, transplant characteristics and transplant-related complications were compared between the DAH cases and the controls. The duration from DAH to death, the duration of hospitalization, intensive care unit (ICU) administration and the need for mechanical ventilation, the improvements in symptom and oxygenation index, secondary infections and transplantation survival were compared between the high- and low-dose corticosteroid groups using the $\chi^{2}$ and Fisher's exact tests for dichotomous variables and a $t$ test for continuous variables. The factors with a $p$ value $<0.1$ as determined by univariate logistic analysis were included in the multivariate logistic regression analysis, and 
the factors with a p value $<0.05$ were considered as being independently associated with the occurrence of DAH. All of the reported $p$ values were based on two-sided tests. The data analyses were conducted using the SPSS software package (SPSS Inc., Chicago, Ill., USA).

\section{Results}

\section{Characteristics of the DAH Cases}

The mean age at the time of presentation for the 22 DAH cases was 30.4 years $( \pm 12.9)$, and the mean time to presentation was 7.8 months $( \pm 8.1)$ post-HSCT. Dyspnea was the most common symptom (95.5\%) and 20 patients had a body temperature higher than $37.2^{\circ} \mathrm{C}$ at the time of diagnosis. All of the patients developed bilateral, diffuse radiographic abnormalities involving all lung zones and pleural effusions were found in 10 patients. A total of 20 patients received corticosteroids. Twelve patients needed to be admitted to the ICU because of disease aggravation and all of them received invasive mechanical ventilator support.

Symptoms improved in 8 out of $11(72.7 \%)$ patients in the high-dose MP group compared to 3 out of $11(27.3 \%)$ patients in the low-dose MP group ( $\mathrm{p}=0.033)$, but secondary infection was more common in the high-dose MP group (36.4 vs. 9.1\%, $\mathrm{p}=0.311$ ). The duration from DAH to death, the ratio of ICU administration and mechanical ventilation, the duration of ICU administration and mechanical ventilation, the duration of hospitalization, the improvement in the oxygenation index and the transplantation survival were all comparable between the high- and low-dose MP groups (data not shown).

\section{Pre-HSCT Comorbidities}

HCT-CI scores, which were used to evaluate the severity of pre-HSCT comorbidities, were comparable between DAH cases and controls (table 1).

\section{Pre-HSCT Chemotherapeutic Exposure and Conditioning Regimens}

The pre-HSCT cyclophosphamide exposure and the cumulative cyclophosphamide dose were both significantly higher among the DAH cases compared to the controls and the proportion of patients who received a cumulative cyclophosphamide dose of $\geq 5 \mathrm{~g} / \mathrm{m}^{2}$ was also higher among the DAH cases ( 45.5 vs. $21.2 \%, \mathrm{p}=0.027$ ). The number of cycles of pre-HSCT chemotherapy, preHSCT exposure of the other chemotherapeutic agents and the types of conditioning-related exposures were

High-Dose Cyclophosphamide

Associated with DAH

Table 3. Characteristics of severe transplant-related complications

DAH cases $^{\mathrm{a}}$ Controls $^{\mathrm{a}} \mathrm{p}$

$(\mathrm{n}=22) \quad(\mathrm{n}=66)$

\begin{tabular}{lccc}
$\begin{array}{l}\text { Transplant-related complication by type } \\
\text { Acute GVHD }\end{array}$ & & \\
$\quad$ Grades III-IV & $11(50.0)$ & $19(28.8)$ & $0.069^{*}$ \\
$\begin{array}{l}\text { Chronic GVHD } \\
\quad \text { Categories moderate to severe }\end{array}$ & $8(36.4)$ & $20(30.3)$ & 0.597 \\
$\begin{array}{l}\text { Engraftment syndrome } \\
\text { Thrombotic microangiopathy }\end{array}$ & $1(4.5)$ & $3(4.5)$ & 1.000 \\
$\begin{array}{l}\text { Post-transplantation } \\
\quad \text { lymphoproliferative disease }\end{array}$ & $3(13.6)$ & $1(1.5)$ & 0.153 \\
$\begin{array}{l}\text { Transplant-related complication by number } \\
\text { No complications }\end{array}$ & $3(13.6)$ & $26(39.4)$ & $0.097^{*}$ \\
$\begin{array}{l}1 \text { complication } \\
\geq 2 \text { complications }\end{array}$ & $11(50.0)$ & $26(39.4)$ & \\
& $8(36.4)$ & $14(21.2)$ & \\
\hline
\end{tabular}

Values are presented as $\mathrm{n}(\%) .{ }^{*} \mathrm{p}<0.1$.

${ }^{a}$ Group matching criteria included year of HSCT ( \pm 2 years) and duration of follow-up ( \pm 2 months).

comparable between the DAH cases and the controls (table 2).

\section{Severe Transplant-Related Complications}

Severe acute GVHD ( $\geq$ grade III), post-transplantation lymphoproliferative disease and two or more severe transplant-related complications were more frequently observed in patients with DAH. No patients suffered from hepatic sinusoidal obstruction syndrome among the DAH cases and the controls (table 3).

\section{Risk Factors for DAH}

In the univariate logistic regression, pre-HSCT cyclophosphamide exposure $\geq 3 \mathrm{~g} / \mathrm{m}^{2}$, the cumulative cyclophosphamide dose $\geq 5 \mathrm{~g} / \mathrm{m}^{2}$ and post-transplantation lymphoproliferative disease were associated with DAH $(\mathrm{p}<0.1)$. In the multivariate logistic regression analysis, the cumulative cyclophosphamide dose was the only risk factor that was significantly and independently associated with DAH $(\mathrm{OR}=3.4,95 \% \mathrm{CI} 1.1-10.2, \mathrm{p}=0.030)$ and the other factors were eliminated (table 4).

\section{Pre-HSCT Cyclophosphamide Exposure and}

Diagnosis

Cyclophosphamide was an unusual drug to be used in AML and was only used among the AML patients with 
Table 4. Univariate and multivariate analysis of risk factors for $\mathrm{DAH}$

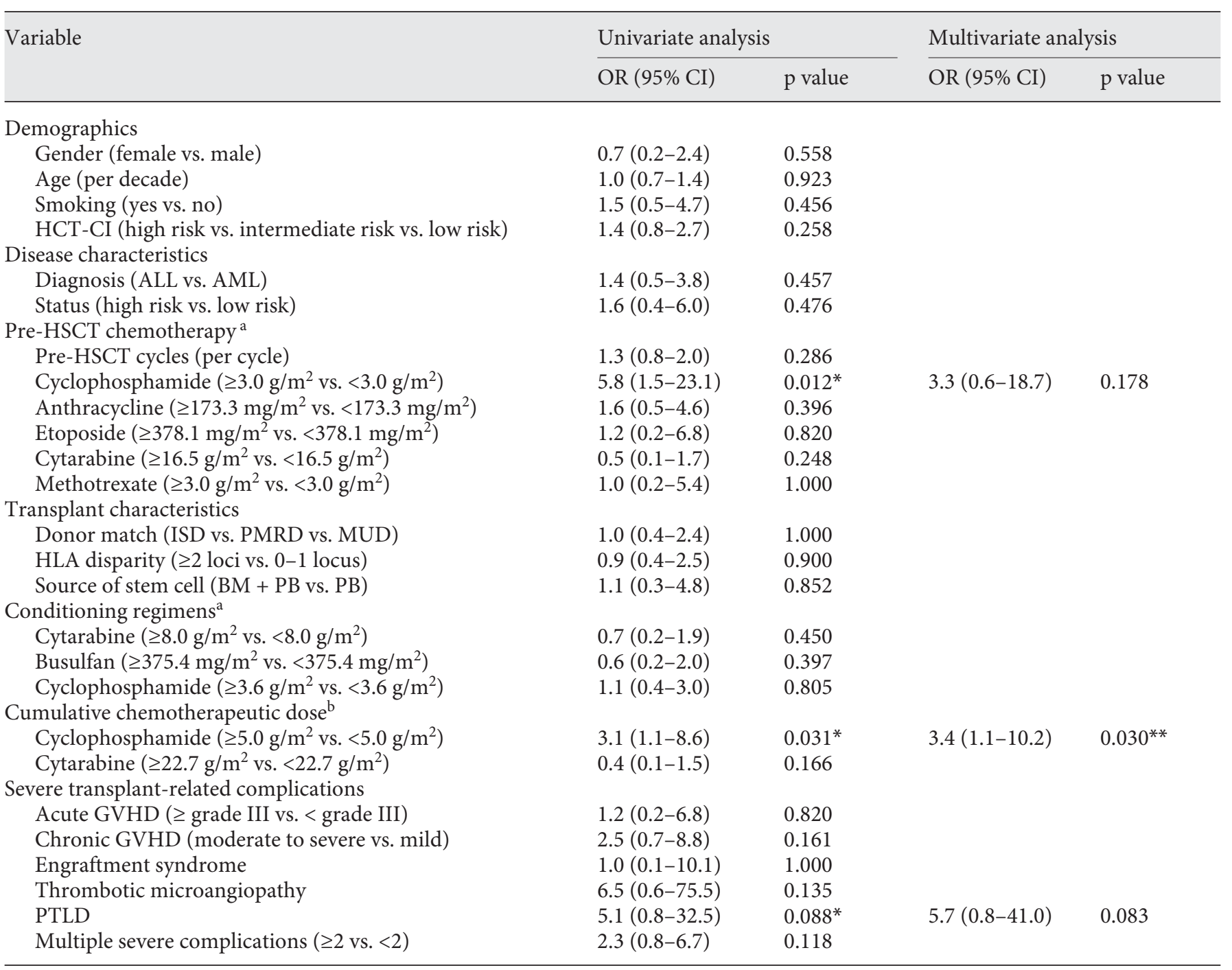

${ }^{*} \mathrm{p}<0.1$ in univariate analysis; ${ }^{* *} \mathrm{p}<0.05$ in multivariate analysis.

$\mathrm{BM}=$ Bone marrow stem cell; HCT-CI = hematopoietic cell transplantation-specific comorbidity index; HLA = human leukocyte antigen; $\mathrm{MUD}=$ matched unrelated donor; PTLD = post-transplantation lymphoproliferative disease.

a The 75th percentile was used as the cutoff point.

the expression of lymphoid-associated antigens in our institute. A total of 24 patients received a cumulative cyclophosphamide dose of $\geq 5 \mathrm{~g} / \mathrm{m}^{2}$ in the ALL group, compared to no patients in the AML group.

\section{Outcomes of DAH}

Of the 22 cases, 19 (86.4\%) patients died with DAH the most common cause of death (73.7\%), followed by infection (21.1\%). Among the 11 patients who showed improvement in symptoms, 6 suffered symptom deterioration again and died of DAH (4 in the high-dose MP group and 2 in the low-dose MP group). The mean duration from the time of improvement to deterioration was 5 days $( \pm 8)$. Among the other 5 patients, 2 died, 1 of infection and the other of post-transplantation lymphoproliferative disease. Only 3 patients survived ( 2 in the high-dose MP group and 1 in the low-dose MP group). 


\section{Discussion}

A total of 22 out of 597 acute leukemia patients developed DAH after allo-HSCT, $86.4 \%$ of them died with DAH the most common cause of death. This result demonstrates that DAH is a rare but severe pulmonary complication of allo-HSCT. We firstly showed that high cumulative cyclophosphamide dose $\left(\geq 5 \mathrm{~g} / \mathrm{m}^{2}\right)$ was independently associated with DAH after allo-HSCT. Although high-dose corticosteroids could transiently improve symptoms, it may not alter the poor outcome associated with this syndrome.

Cyclophosphamide is an alkylating cytotoxic agent that is widely used to treat hematological malignancies. Lung injury is one of its severe toxic side effects [21-23]. The incidence of cyclophosphamide-induced lung disease is $1 \%$ [24] and symptoms may begin several weeks to several years after the initial administration of this drug $[21,22,24,25]$. Cyclophosphamide can influence the morphology and function of type 2 pneumocytes and lead to fibroblast proliferation and fibrosis, endothelial swelling, and septal edema, which are consistent with severe injury involving all alveolar components [21, 24]. These initial injuries may be implicated in the pathogenesis of DAH in the allo-HSCT recipients [9]. Jiang et al. [26] reported that 2 patients with hematological malignancies suffered from DAH 1 month and several hours, respectively, after the administration of cyclophosphamide, but no research was conducted to evaluate the association between this agent and DAH after allo-HSCT. In our study, we found that pre-HSCT cyclophosphamide exposure and the cumulative cyclophosphamide dose were both significantly higher among the DAH cases compared to the controls, and patients that received higher cumulative doses of cyclophosphamide were at greater risk for developing DAH. Due to the poor outcome of $\mathrm{DAH}$, conditioning regimens that use a lower dose of cyclophosphamide or without cyclophosphamide may be more appropriate for patients with high pre-HSCT cyclophosphamide exposure, but the dose of cyclophosphamide and alternative regimens should be further evaluated. On the other hand, because cyclophosphamide is the usual drug used in ALL, this result suggested that ALL patients might be at a higher risk of developing DAH after allo-HSCT.

Because inflammation and cytokine release may mediate the development of DAH, systemic corticosteroids are widely used to treat DAH. However, the high-dose corticosteroid strategy remains controversial. Some studies found that DAH is reversible by treatment with high-dose corticosteroid [4, 27, 28]. Nevertheless, Uchiyama and Ikeda [29] reported that DAH might be successfully treated using a relatively low-dose corticosteroid (prednisolone at $1 \mathrm{mg} / \mathrm{kg} /$ day). Other studies found that the use of high-dose methylprednisolone was not associated with a significant improvement in survival in patients with DAH $[1,2,3,8]$. In our study, to more precisely evaluate the dose of corticosteroid, we recalculated the dose that they received based on body surface area. Although a transient improvement in symptoms was more common in the high-dose MP group, most of the patients deteriorated soon after and the poor outcome was similar to that of the low-dose MP group. It has been suggested that not only nonspecific inflammation but also some infectious agents, which cannot be detected by our current microbiological tests, may contribute to DAH [2]. We also found that secondary infections were more common in the high-dose MP group, which would be another factor negatively influencing the effect of high-dose corticosteroid therapy, and prevention or preemptive therapy for infections should be aggressively sought.

Majhail et al. [1] reported that age is a risk factor for DAH after allo-HSCT, and the risk increased 1.2- to 1.5fold for every 10 years of age (median age was 40 years). It has been suggested that the lungs of the older patients might be more susceptible to the conditioning regimens and transplant-related complications. In our study, we did not observe any significant correlation between age and DAH after allo-HSCT. It is possible that our patients were younger. Nevertheless, Ho et al. [5] found that a proportion of subjects who were older than 50 years at HSCT had no significant difference between DAH cases and controls, and Gupta et al. [30] found that there was no correlation between the age at HSCT and the incidence of DAH (median age was 50 years). Thus, the age difference might have a limited effect on DAH, but the effect cannot be completely excluded.

Some studies have reported that there is an association between severe transplant-related complications (such as severe acute GVHD and thrombotic microangiopathy) and DAH $[1,9,10]$. In our study, we observed that severe acute GVHD, post-transplantation lymphoproliferative disease and multiple severe transplant-related complications occurred predominantly in DAH cases, but the small sample sizes of patients with DAH limited our ability to further describe the contribution of transplant-related complications in at-risk survivors. The trend suggested that the difference would have been more significant if more patients were studied and fu- 
ture studies involving multicenter study designs would help to provide more evidence for the correlation between severe transplant-related complications and DAH.

In summary, we report that the high cumulative cyclophosphamide dose was independently associated with DAH after allo-HSCT and high-dose corticosteroid therapy may not alter the poor outcome associated with this syndrome. These results may help us to identify the patients who are at higher risk of developing DAH after allo-HSCT and to select a more appropriate therapeutic strategy.

\section{Acknowledgments}

This work was partly supported by the National High Technology Research and Development Program of China (Program 863; Grant No. 2011AA020105) and the National Natural Science Foundation of China (Grant No. 30971292). T. Suja and A. Quyen, who are medical writers supported by funding from American Journal Experts, provided an editing service to the authors during preparation of the manuscript.

\section{Financial Disclosure and Conflicts of Interest}

The authors declare no conflicts of interest.

\section{References}

1 Majhail NS, Parks K, Defor TE, Weisdorf DJ: Diffuse alveolar hemorrhage and infectionassociated alveolar hemorrhage following hematopoietic stem cell transplantation: related and high-risk clinical syndromes. Biol Blood Marrow Transplant 2006;12:10381046.

2 Lewis ID, DeFor T, Weisdorf DJ: Increasing incidence of diffuse alveolar hemorrhage following allogeneic bone marrow transplantation: cryptic etiology and uncertain therapy. Bone Marrow Transplant 2000;26:539-543.

3 Afessa B, Tefferi A, Litzow MR, Peters SG: Outcome of diffuse alveolar hemorrhage in hematopoietic stem cell transplant recipients. Am J Respir Crit Care Med 2002;166 1364-1368.

-4 Raptis A, Mavroudis D, Suffredini A, Molldrem J, Rhee FV, Childs R, Phang S, Barrett A: High-dose corticosteroid therapy for diffuse alveolar hemorrhage in allogeneic bone marrow stem cell transplant recipients. Bone Marrow Transplant 1999;24:879-883.

5 Ho VT, Weller E, Lee SJ, Alyea EP, Antin JH, Soiffer RJ: Prognostic factors for early severe pulmonary complications after hematopoietic stem cell transplantation. Biol Blood Marrow Transplant 2001;7:223-229.

-6 Wanko SO, Broadwater G, Folz RJ, Chao NJ: Diffuse alveolar hemorrhage: retrospective review of clinical outcome in allogeneic transplant recipients treated with aminocaproic acid. Biol Blood Marrow Transplant 2006;12:949-953.

7 Witte RJ, Gurney JW, Robbins RA, Linder J, Rennard SI, Arneson M, Vaughan WP, Reed EC, Dicke KA: Diffuse pulmonary alveolar hemorrhage after bone marrow transplantation: radiographic findings in 39 patients. AJR Am J Roentgenol 1991;157:461-464.
8 Ben-Abraham R, Paret G, Cohen R, Szold O, Cividalli G, Toren A, Nagler A: Diffuse alveolar hemorrhage following allogeneic bone marrow transplantation in children. Chest 2003;124:660-664.

-9 Afessa B, Tefferi A, Litzow MR, Krowka MJ, Wylam ME, Peters SG: Diffuse alveolar hemorrhage in hematopoietic stem cell transplant recipients. Am J Respir Crit Care Med 2002;166:641-645.

10 Srivastava A, Gottlieb D, Bradstock KF: Diffuse alveolar haemorrhage associated with microangiopathy after allogeneic bone marrow transplantation. Bone Marrow Transplant 1995;15:863-867.

11 Wacholder S, Silverman DT, McLaughlin JK, Mandel JS: Selection of controls in case-control studies. III. Design options. Am J Epidemiol 1992;135:1042-1050.

12 Huang XJ, Liu DH, Liu KY, Xu LP, Chen H, Han W, Chen YH, Wang JZ, Gao ZY, Zhang YC, Jiang Q, Shi HX, Lu DP: Haploidentical hematopoietic stem cell transplantation without in vitro $\mathrm{T}$-cell depletion for the treatment of hematological malignancies. Bone Marrow Transplant 2006;38:291-297.

13 Sakr L, Dutau H: Massive hemoptysis: an update on the role of bronchoscopy in diagnosis and management. Respiration 2010;80:3858.

14 Sakata-Yanagimoto M, Kanda Y, Nakagawa M, Asano-Mori Y, Kandabashi K, Izutsu K, Imai Y, Hangaishi A, Kurokawa M, Tsujino S, Ogawa S, Chiba S, Motokura T, Hirai H: Predictors for severe cardiac complications after hematopoietic stem cell transplantation. Bone Marrow Transplant 2004;33: 1043-1047.

15 Sorror ML, Maris MB, Storb R, Baron F, Sandmaier BM, Maloney DG, Storer B: Hematopoietic cell transplantation (HCT)-specific comorbidity index: a new tool for risk assessment before allogeneic HCT. Blood 2005;106:2912-2919.
16 Przepiorka D, Weisdorf D, Martin P, Klingemann HG, Beatty P, Hows J, Thomas ED: 1994 consensus conference on acute GVHD grading. Bone Marrow Transplant 1995; 15: 825-828.

17 Filipovich AH, Weisdorf D, Pavletic S, Socie G, Wingard JR, Lee SJ, Martin P, Chien J, Przepiorka D, Couriel D, Cowen EW, Dinndorf P, Farrell A, Hartzman R, HensleeDowney J, Jacobsohn D, McDonald G, Mittleman B, Rizzo JD, Robinson M, Schubert M, Schultz K, Shulman H, Turner M, Vogelsang G, Flowers ME: National Institutes of Health consensus development project on criteria for clinical trials in chronic graftversus-host disease. I. Diagnosis and staging working group report. Biol Blood Marrow Transplant 2005;11:945-956.

18 Batts ED, Lazarus HM: Diagnosis and treatment of transplantation-associated thrombotic microangiopathy: real progress or are we still waiting? Bone Marrow Transplant 2007;40:709-719.

19 Gulley ML, Tang W: Using Epstein-Barr viral load assays to diagnose, monitor, and prevent posttransplant lymphoproliferative disorder. Clin Microbiol Rev 2010;23:350-366.

20 Helmy A: Review article: updates in the pathogenesis and therapy of hepatic sinusoidal obstruction syndrome. Aliment Pharmacol Ther 2006;23:11-25.

21 Aronchick JM, Gefter WB: Drug-induced pulmonary disorders. Semin Roentgenol 1995;30:18-34.

22 Limper AH: Chemotherapy-induced lung disease. Clin Chest Med 2004;25:53-64.

23 Camus P, Fanton A, Bonniaud P, Camus C, Foucher P: Interstitial lung disease induced by drugs and radiation. Respiration 2004;71: 301-326. 
-24 Hamada K, Nagai S, Kitaichi M, Jin G, Shigematsu M, Nagao T, Sato A, Mishima M: Cyclophosphamide-induced late-onset lung disease. Intern Med 2003;42:82-87.

25 Malik SW, Myers JL, DeRemee RA, Specks U: Lung toxicity associated with cyclophosphamide use: two distinct patterns. Am J Respir Crit Care Med 1996;154:1851-1856.

-26 Jiang Q, Yang SM, Jiang B, Lu BB, Huang XJ, Wang DB: Diffuse alveolar hemorrhage as a complication in patients with hematologic malignancies after chemotherapy: report of two cases and literature review. Zhonghua Xue Ye Xue Za Zhi 2007;28:230-234.
27 Metcalf JP, Rennard SI, Reed EC, Haire WD, Sisson JH, Walter T, Robbins RA: Corticosteroids as adjunctive therapy for diffuse alveolar hemorrhage associated with bone marrow transplantation. University of Nebraska Medical Center Bone Marrow Transplant Group. Am J Med 1994;96:327-334.

28 Haselton DJ, Klekamp JG, Christman BW, Barr FE: Use of high-dose corticosteroids and high-frequency oscillatory ventilation for treatment of a child with diffuse alveolar hemorrhage after bone marrow transplantation: case report and review of the literature. Crit Care Med 2000;28:245-248.
9 Uchiyama M, Ikeda T: Diffuse alveolar hemorrhage after unrelated cord blood transplantation. Bone Marrow Transplant 2010; 45:789-790.

30 Gupta S, Jain A, Warneke CL, Gupta A, Shannon VR, Morice RC, Onn A, Jimenez CA, Bashoura L, Giralt SA, Dickey BF, Eapen GA: Outcome of alveolar hemorrhage in hematopoietic stem cell transplant recipients. Bone Marrow Transplant 2007;40:71-78. 